\title{
A Case of Right Paratracheal Ectopic Thyroid, Mimicking Metastasis on CT and ${ }^{18}$ F-FDG PET CT
}

\author{
Su Young Kim \\ Department of Radiology, Inje University Ilsan Paik Hospital, Seoul, South Korea \\ Email: sykim@paik.ac.kr
}

Received May 17, 2013; revised June 16, 2013; accepted June 30, 2013

Copyright (C) 2013 Su Young Kim. This is an open access article distributed under the Creative Commons Attribution License, which permits unrestricted use, distribution, and reproduction in any medium, provided the original work is properly cited.

\begin{abstract}
Mediastinal ectopic thyroid is quite rare, occurring in less than $1 \%$ of all ectopic thyroids. Frequent location of mediastinal ectopic thyroid in reported cases is anterosuperior mediastinum. CT findings of ectopic thyroid can be various and are dependent on several factors, such as its iodine content, the extent of tissue degeneration, and the presence of colloid cysts. This is a case of right paratracheal ectopic thyroid with nodular hyperplasia, mimicking metastatic nodal mass on both CT and ${ }^{18}$ F-FDG PET CT in a patient with colon cancer.
\end{abstract}

Keywords: Ectopic Thyroid; Computed Tomography; ${ }^{18}$ F-FDG PET CT

\section{Introduction}

Ectopic thyroid is a rare entity and is the result of abnormal migration of the thyroid gland during its passage from the floor of the primitive foregut to pre-tracheal position. Most frequent location of ectopic thyroid is at the region of the foramen cecum and it accounts for about $90 \%$ of the abnormalities. Primary mediastinal ectopic thyroid is quite rare and is encountered in less than $1 \%$ of all goiters. CT findings of ectopic thyroid can be various and are dependent on several factors, such as its iodine content, the extent of tissue degeneration, and the presence of colloid cysts. This is a case of ectopic thyroid occurring in the mediastinal paratracheal area, which mimicked metastatic nodal mass on CT and 18FFDG PET CT scan in a patient with history of colon cancer.

\section{Case Report}

A 67-year-old man with a history of colon cancer was referred to our thoracic surgery department for operation of incidentally detected mediastinal mass on FDG-PET scan. The mass showed mild increase in size during 3month follow-up. On FDG-PET CT, the mass showed diffuse strong FDG uptake with maximum SUV of 8 (Figure 1). Preoperative chest CT scan was performed with a 320 channel MDCT (Aquilion ONE, Toshiba, Tokyo, Japan). The images were obtained with a $2.5-\mathrm{mm}$ slice thickness for the axial scan and the coronal images

Copyright (C) 2013 SciRes. were reconstructed automatically on the console. The contrast enhanced images were routinely obtained 40 seconds after initiating the injection of contrast medium at a rate of $3 \mathrm{ml} / \mathrm{s}$. On precontrast scan, the mass showed heterogenous in attenuation with peripheral dotted calcifications (Figure 2). The central portion of the mass was low attenuated and the peripheral portion was almost isoattenuated with muscles of the chest wall (Figure 2). The enhanced scan revealed the well-demarcated peripheral strong enhancing mass with irregular low attenuating central area (Figures 3 and 4). Based on these findings of both CT and PET scans, the differential diagnosis included metastatic necrotic lymph node, tuberculous lymphadenitis, neurogenic tumor and ectopic thymic tumor. Surgical resection was done and the mass was completely separated with normal thyroid. On gross specimen, the multilobulated mass was partially encapsulated and the cut surface was reddish soft tissue with hemorrhagic appearance. There was no necrotic change within the mass. The histopathologic examination confirmed ectopic thyroid with nodular hyperplasia (Figure 5). The patient was asymptomatic preoperatively and is still euthyroid postoperatively.

\section{Discussion}

Primary intrathoracic goiter, which is named alternately as ectopic goiter is defined as being intrathoracic bulk of thyroid tissue receiving its blood supply from mediastinal vessels and being unconnected to the thyroid in the 


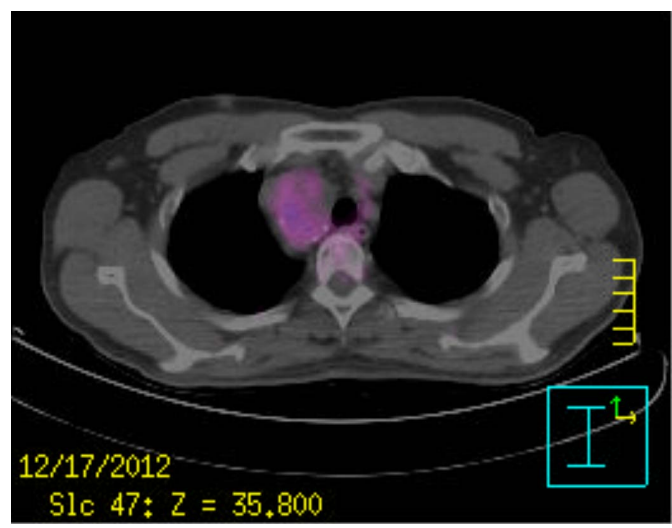

Figure 1. On fusion image of 18F-FDG PET CT, the mass shows diffuse strong FDG uptake and the maximum SUV was 8.

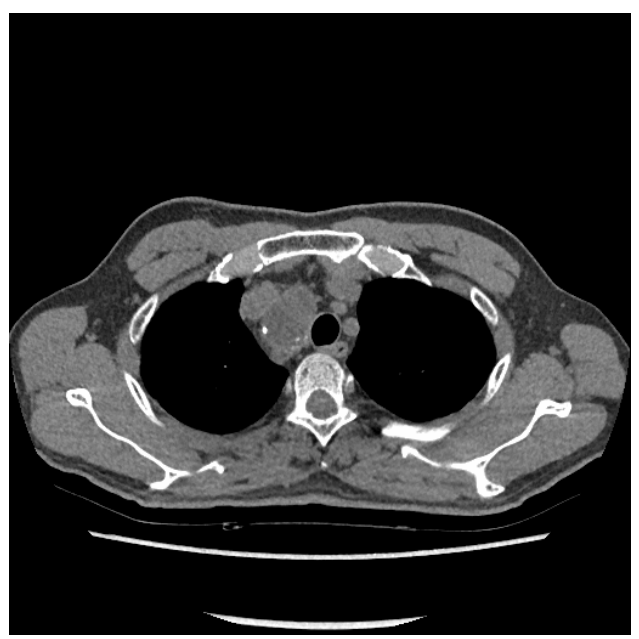

Figure 2. Pre contrast CT scan demonstrates well-defined right paratracheal mass, which is centrally low attenuated and peripherally almost iso-attenuated with muscles of the chest wall. Multiple peripheral calcifications are seen.

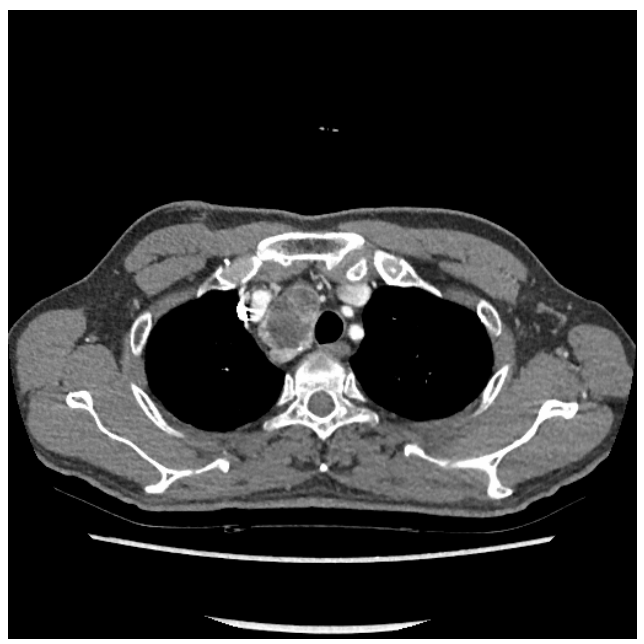

Figure 3. Enhnaced axial and coronal CT scans depict the peripheral strong enhancing mass with irregular low attenuating central area.

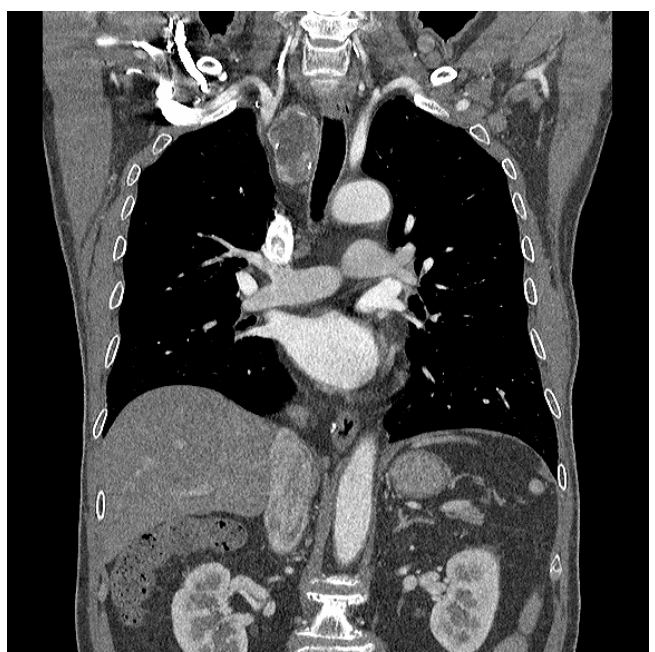

Figure 4. Enhnaced axial and coronal CT scans depict the peripheral strong enhancing mass with irregular low attenuating central area.

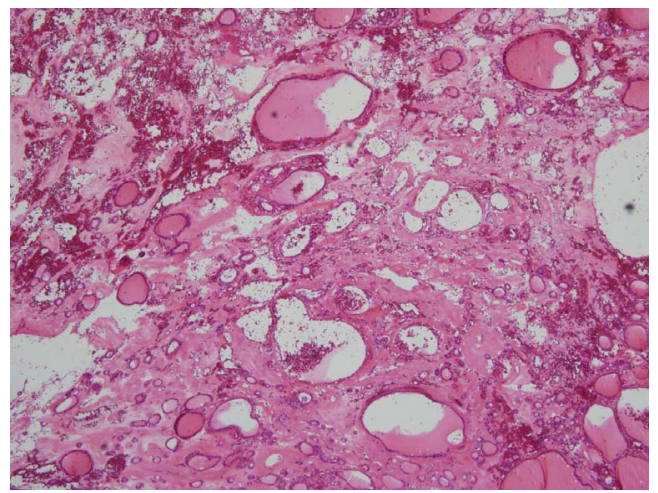

Figure 5. On microscopic examination (original magnification $\times \mathbf{2 0 0}$ ), the mass was composed of colloid distended follicles intermixed with foci of hyperplasia and separated by dense hyalinizing bands of fibrous tissue. These findings were compatible with a multinodular goiter.

normal cervical location [1,2]. True ectopic thyroid in the thorax are encountered in less than $1 \%$ of all intarthoracic goiter $[3,4]$. Ectopic thyroid tissue is the result of aberrant embryogenesis of the thyroid gland during its passage from the floor of the primitive foregut to its pretracheal position. According to autopsy studies, the prevalence ranges between $7 \%$ and $10 \%[5,6]$. Most frequent location of ectopic thyroid tissue is the base of the tongue and termed "lingual thyroid". It accounts for about $90 \%$ of the reported cases [7]. Only 10\% locate in the cervical region. Intrathoracic ectopic thyroid is rare location of ectopic thyroid and has been reported in mediastinum, mostlyanterosuperior mediastinum, lungs, trachea and heart. Other extremely rare locations of ectopic thyroid reported in the literatures are subdiaphragmatic areas, involving ovary, adrenal gland, gallbladder, pancreas, duodenum and mesentery of the small intestine. Intrathoracic ectopic thyroid grow slowly but progres- 
sively, favoring toward the pleural cavity with least resistance $[1,8]$. The mechanism for the growth is not clear, but according to some theories, primary intrathoracic goiters may arise from distant ectopic thyroid tissue resulting from increased TSH production after cervical thyroidectomy [9]. Most patients with ectopic thyroid are clinically and biochemically euthyroid, but hyperthyroidism occur in $10 \%$ to $20 \%$ of patients [10].

On chest computed tomography (CT), ectopic thyroid typically appears a well-demarcated mass. Attenuation of the thyroid tissue depends on its iodine content. On precontrast scan, attenuation of the mass can be higher than that of muscles in the chest wall, but if the iodine content is low, the attenuation can be similar to the muscles [11]. On enhanced scan, the mass usually have intense enhancement and the degree of enhancement is dependent on the extent of tissue degeneration [11,12]. Calcifications are frequently seen and there are areas of low attenuation due to colloid cysts [12,13]. Radioactive iodine scan using I-131 or I-123 may be useful in diagnosis of ectopic thyroid in most patients, but a negative scan does not exclude the diagnosis because lack of iodine concentration by ectopic thyroid is possible if there is extensive necrosis or carcinoma. But experience on this study is limited [14]. The normal thyroid shows very low uptake on ${ }^{18}$ F-FDG PET scan and usually not visualized on the scan. Focal or diffuse uptake of thyroid can be seen in various benign and malignant conditions, including thyroid carcinoma, metastatic tumor, thyroiditis, multinodular goiter and nodular hyperplasia [15]. Acute suppurative thyroiditis may occur in intrathoracic ectopic thyroid [16] and cancer can occur in $3 \%$ to $10 \%$ of intrathoracic ectopic thyroid $[2,17]$. Although there is no consensus for the optimal therapeutic strategy, surgical treatment can be considered based on some parameters, such as size, local symptoms, and complication of the mass (ulceration, bleeding, cystic degeneration, malignant transformation) $[6,18]$. In asymptomatic and euthyroid cases, only regular follow-up is recommended.

In conclusion, despite of its rarity, author suggests that radiologists should be aware of its variable radiologic features and the possibility of ectopic thyroid should be considered in differential diagnosis of well-demarcated heterogenous enhancing mediastinal mass.

\section{Acknowledgements}

This paper was supported by a 2011 scientific research grant from Inje University.

\section{REFERENCES}

[1] S. Madjar and D. Weissberg, "Retrosternal Goiter," Chest, Vol. 108, No. 1, 1995, pp. 78-82. doi:10.1378/chest.108.1.78
[2] M. L. White, G. M. Doherty and P. G. Gauger, "Evidence-Based Surgical Management of Substernal Goiter," World Journal of Surgery, Vol. 32, No. 7, 2008, pp. 1285-1300. doi:10.1007/s00268-008-9466-3

[3] T. C. Gamblin, G. R. Jennings, D. B. Christie 3rd, W. M. Thompson Jr. and M. L. Dalton, "Ectopic Thyroid," The Annals of Thoracic Surgery, Vol. 75, No. 6, 2003, pp. 1952-1953. doi:10.1016/S0003-4975(03)00007-9

[4] J. Sand, E. Pehkonen, J. Mattila, S. Seppanen and J. Salmi, "Pulsating Mass at the Sternum: A Primary Carcinoma of Ectopic Mediastinal Thyroid," The Journal of Thoracic and Cardiovascular Surgery, Vol. 112, No. 3, 1996, pp. 833-835.

[5] E. Kousta, K. Konstantinidis, C. Michalakis, M. Vorias, G. Sambalis, M. Georgiou and G. E. Theodoropoulos, "Ectopic Thyroid Tissue in the Lower Neck with a Coexisting Normally Located Multinodular Goiter and Brief Literature Review,” Hormones (Athens), Vol. 4, No. 4, 2005, pp. 231-234.

[6] J. A. Bersaneti, R. D. Silva, R. R. Ramos, M. M. Matsushita and L. R. Souto, "Ectopic Thyroid Presenting as a Submandibular Mass," Head and Neck Pathology, Vol. 5, No. 1, 2011, pp. 63-66. doi:10.1007/s12105-010-0209-Z

[7] S. Basaria, W. H. Westra and D. S. Cooper, "Ectopic Lingual Thyroid Masquerading as Thyroid Cancer Metastases," The Journal of Clinical Endocrinology \& Metabolism, Vol. 86, No. 1, 2001, pp. 392-395. doi:10.1210/jc.86.1.392

[8] J. D. Rives, "Mediastinal Aberrant Goiter," Annals of Surgery, Vol. 126, No. 5, 1947, pp. 797-810. doi:10.1097/00000658-194711000-00012

[9] P. Vadasz and L. Kotsis, "Surgical Aspects of 175 Mediastinal Goiters,” European Journal Cardio-Thoracic Surgery, Vol. 14, No. 4, 1998, pp. 393-397.

[10] R. Prakash, N. Lakshmipathi, A. Jena, V. Behari and M. K. Chopra, "Hyperthyroidism Caused by a Toxic Intrathoracic Goiter with a Normal-Sized Cervical Thyroid Gland,” Journal of Nuclear Medicine, Vol. 27, No. 9, 1986, pp. 1423-1427.

[11] H. S. Glazer, P. L. Molina, M. J Siegel and S. S. Sagel, "High-Attenuation Mediastinal Masses on Unenhanced CT,” American Journal of Roentgenology, Vol. 156, No. 1, 1991, pp. 45-50. doi:10.2214/ajr.156.1.1898569

[12] J. A. Buckley and P. Stark, "Intrathoracic Mediastinal Thyroid Goiter: Imaging Manifestations,” American Journal of Roentgenology, Vol. 173, No. 2, 1999, pp. 471475. doi:10.2214/ajr.173.2.10430156

[13] K. L. Chakraborti, G. Sripathy and T. Sekhri, "Imaging in a Case of Thyrotoxicosis" Indian Journal of Nuclear Medicine, Vol. 20, No. 1, 2005, pp. 14-16.

[14] C. N. Foroulis, K. S. Rammos, M. N. Sileli and C. Papakonstantinou, "Primary Intrathoracic Goiter: A Rare and Potentially Serious Entity,” Thyroid, Vol. 19, No. 3, 2009, pp. 213-218.

[15] T. Y. Kim, W. B. Kim, J. S. Ryu, G Gong, S. J. Hong and Y. K. Shong, “18F-Fluorodeoxyglucose Uptake in Thyroid from Positron Emission Tomogram (PET) for Evaluation in Cancer Patients: High Prevalence of Malignancy 
in Thyroid PET Incidentaloma,” Laryngoscope, Vol. 115, No. 6, 2005, pp. 1074-1078.

[16] C. R. McHenry, F. Paras Jr., Y. C. Liu and M. Rodgers, "Acute Suppurative Thyroiditis in a Primary Intrathoracic Goiter,” Endocrine Practice, Vol. 3, No. 1, 1997, pp. 1418.

[17] M. Nervi, P. Iacconi, C. Spinelli, A. Janni and P. Miccoli, "Thyroid Carcinoma in Intrathoracic Goiter," Langen- beck's Archives of Surgery, Vol. 383, No. 5, 1998. pp. 337-339. doi:10.1007/s004230050144

[18] G. Noussios, P. Anagnostis, D. G. Goulis, D. Lappas and K. Natsis, "Ectopic Thyroid Tissue: Anatomical, Clinical, and Surgical Implications of a Rare Entity,” European Journal of Endocrinology, Vol. 165, No. 3, 2011, pp. 375-382. 